Bull. Soc. math. France

134 (4), 2006, p. 451-473

\title{
STEINNESS OF BUNDLES WITH FIBER A REINHARDT BOUNDED DOMAIN
}

\author{
By Karl Oelueklaus \& Dan Zaffran
}

\begin{abstract}
Let $E$ denote a holomorphic bundle with fiber $D$ and with basis $B$. Both $D$ and $B$ are assumed to be Stein. For $D$ a Reinhardt bounded domain of dimension $d=2$ or 3 , we give a necessary and sufficient condition on $D$ for the existence of a non-Stein such $E$ (Theorem 1 ); for $d=2$, we give necessary and sufficient criteria for $E$ to be Stein (Theorem 2). For $D$ a Reinhardt bounded domain of any dimension not intersecting any coordinate hyperplane, we give a sufficient criterion for $E$ to be Stein (Theorem 3).

RÉSumÉ (Fibrés de Stein à fibre un domaine de Reinhardt borné)

Soit $E$ un fibré holomorphe à fibre $D$ et base $B$. On suppose que $D$ et $B$ sont de Stein. Si $D$ est un domaine de Reinhardt borné de dimension 2 ou 3, on donne une condition nécessaire et suffisante sur $D$ pour l'existence d'un tel fibré $E$ qui ne soit pas Stein (Théorème 1) ; pour $d=2$ on donne des conditions nécessaires et suffisantes pour que $E$ soit de Stein (Théorème 2). Si $D$ est un domaine de Reinhardt de dimension quelconque qui n'intersecte pas les hyperplans de coordonnées, on donne un critère suffisant pour que $E$ soit de Stein.
\end{abstract}

Texte reçu le 20 juillet 2004, accepté le $1^{\text {er }}$ février 2005.

Karl Oeljeklaus, Université de Provence, CMI et LATP (UMR CNRS 6632), 39 rue JoliotCurie, F-13453 Marseille Cedex 13 • E-mail : karl.oeljeklaus@cmi.univ-mrs.fr Dan Zaffran, Cornell University, Malott Hall, Ithaca NY 14853, U.S.A.

E-mail : zaffran@math.cornell.edu - Current affiliation: Fudan University, Shanghai 2000 Mathematics Subject Classification. - 32E10, 32A07, 32L05.

Key words and phrases. - Holomorphic fiber bundle, Stein manifold, bounded Reinhardt domain, Serre problem.

The second named author wishes to warmly thank A. Huckleberry for his hospitality in Bochum in 2001, where part of this work was done. We thank as well S. Chase, I. Chatterji, J. Hubbard, P. Liardet and K. Wortman for useful conversations. This work has been done independently from [9].

BUlletin DE LA SOCIÉtÉ MATHÉMATIQUe DE FRANCE (C) Société Mathématique de France

$0037-9484 / 2006 / 451 / \$ 5.00$ 


\section{Introduction and notations}

Stein manifolds can be characterized by the fact that they holomorphically embed in $\mathbb{C}^{N}$ for some $N$. From that point of view it is obvious that if $F$ and $B$ are Stein manifolds, then the product $E=F \times B$ is Stein. More generally, take a fiber bundle $E$ with fiber $F$ and with basis $B$, which we shall denote by

$$
E \stackrel{F}{\longrightarrow} B \text {. }
$$

Is such an $E$ necessarily Stein? That question was asked fifty years ago by J.-P. Serre [10], and is often referred to as "the Serre Problem" in the literature.

A counterexample with $F=\mathbb{C}^{2}$ was produced by H. Skoda [16] in 1977. On the other hand, many interesting "positive results" have been obtained (see below).

Following [9], we shall say that a Stein manifold $F$ is of class $\mathcal{S}$, or $F \in \mathcal{S}$ for short, when ${ }^{(1)}$

$$
\text { For any bundle } E \stackrel{F}{\longrightarrow} B \text { with } B \text { Stein, } E \text { is Stein. }
$$

K. Stein [18] proved that if $\operatorname{dim} F=0$, then $F \in \mathcal{S}$. Building on previous work by A. Hirschowitz [5], Y.T. Siu [13] and N. Sibony [12], N. Mok [7] proved that if $\operatorname{dim} F=1$, then $F \in \mathcal{S}$. Skoda's above result can be stated as: $\mathbb{C}^{2} \notin \mathcal{S}$.

In this paper we focus on the case where $F$ is a bounded domain $D \subset \mathbb{C}^{n}$ ("domain" means connected open subset). Several results showing that large classes of bounded domains belong to $\mathcal{S}$ have been proved (cf. [14], [17] and [2]). Nevertheless G. Cœuré and J.-J. Lœeb [1] produced, for each given $A \in \mathrm{SL}_{2}(\mathbb{Z})$ with $|\operatorname{trace} A|>2$, a non Stein bundle

$$
E_{\mathrm{CL}} \stackrel{D_{\mathrm{CL}}}{\longrightarrow} \mathbb{C}^{*}
$$

whose fiber $D_{\mathrm{CL}}$ is a bounded Stein domain subset of $\left(\mathbb{C}^{*}\right)^{2}$. Thus $D_{\mathrm{CL}} \notin \mathcal{S}$. Their $D_{\mathrm{CL}}$ has the following properties (see Figure 1):

$\triangleright D_{\mathrm{CL}}$ has the Reinhardt symmetry, i.e., it is invariant by the map $\left(z_{1}, z_{2}\right) \mapsto$ $\left(\alpha_{1} z_{1}, \alpha_{2} z_{2}\right)$ for any complex numbers $\alpha_{1}$ and $\alpha_{2}$ of modulus 1 ;

$\triangleright D_{\mathrm{CL}}$ has an automorphism $g$ of the form $g\left(z_{1}, z_{2}\right)=\left(z_{1}^{a} z_{2}^{b}, z_{1}^{c} z_{2}^{d}\right)$ with

$$
\left[\begin{array}{ll}
a & b \\
c & d
\end{array}\right]=A
$$

The second named author generalized their construction and gave a better understanding of those bundles. In [20], a key point is the existence of a g-equivariant open dense embedding $D_{\mathrm{CL}} \hookrightarrow \widehat{D}_{\mathrm{CL}}$, where $\widehat{D}_{\mathrm{CL}} \backslash D_{\mathrm{CL}}$ is an infinite chain of rational curves. Roughly speaking, the non-Steinness of $E_{\mathrm{CL}}$ is "explained" by what happens near that infinite chain.

(1) Conveniently enough, that letter honors simultaneously Serre, Sibony, Siu, Skoda, Stehlé and Stein.

TOME $134-2006-\mathrm{N}^{\mathrm{O}} 4$ 
Our goal here is to answer the following "converse" question:

Let $D \subset \mathbb{C}^{n}$, with $n=2$ or 3 , be any bounded Stein Reinhardt domain. Does $D$ belong to $\mathcal{S}$ ?

In other words, does there exist a bundle $E \stackrel{D}{\longrightarrow} B$ with $B$ Stein and $E$ non-Stein? The answer is contained in Theorem 1 below. It reveals that in dimension two, Cœuré-Lœb's examples $D_{\mathrm{CL}}$ (for all $A \in \mathrm{SL}_{2}(\mathbb{Z})$ ) are essentially the only Reinhardt bounded domains not in $\mathcal{S}$ : all other examples are provided by $g$-invariant subdomains of some $D_{\mathrm{CL}}$. Moreover, it is easily checked that the interior of the closure in $\widehat{D}_{\mathrm{CL}}$ of such a subdomain still contains the infinite chain of curves, so from the point of view of [20], it is natural that those domains do not belong to $\mathcal{S}$. Indeed, proofs in [1] and [20] apply almost verbatim to show that they do not belong to $\mathcal{S}$. We shall see that both methods and results become more complicated in dimension three.

We also address the following question:

Given $D$ bounded and Reinhardt not in $\mathcal{S}$ and B Stein, can we give a characterization of the Steinness of a bundle $E \stackrel{D}{\longrightarrow} B$ ?

For a two-dimensional $D$, we give in Theorem 2 both a necessary criterion and a sufficient criterion. For a higher dimensional $D$, we give a partial answer in Theorem 3.

We work throughout the article in the complex category. In other words, all manifolds and maps we deal with are holomorphic. By the word "bundle" we mean a locally trivial holomorphic fiber bundle. We shall also use the notations $\mathcal{O}(E)$ for the set of holomorphic functions on $E$, and $S^{1}$ and $\Delta$ will respectively denote the unit circle and the unit disk in $\mathbb{C}$.

N.B.: Most proofs are postponed until the end of the paper, in Section 4.

We shall make use of several known results about a given bundle $E \stackrel{D}{\longrightarrow} B$ with $B$ Stein and $D \subset \mathbb{C}^{n}$ bounded and Stein. Namely:

$\triangleright E$ is a flat bundle (see [6] or [14]).

That means $E$ can be defined by locally constant transition functions.

All flat bundles can be constructed as follows. Let $\rho$ be a morphism $\pi_{1}(B) \rightarrow$ Aut $(D)$. Such a $\rho$ induces a $\pi_{1}(B)$-action on $D$. Denote by $\widetilde{B}$ the universal cover of $B$, and consider the diagonal action of $\pi_{1}(B)$ on $\widetilde{B} \times D$. Define

$$
E=\frac{\widetilde{B} \times D}{\pi_{1}(B)} .
$$

Then the projection map $\widetilde{B} \times D \rightarrow \widetilde{B}$ induces a map $E \rightarrow B$ that turns $E$ into a bundle with fiber $D$. The structural group $G_{\text {struct }}(E)$ of $E$ is by definition the image of $\rho$. That definition is quite improper because $G_{\text {struct }}(E)$ does not 
only depend on the isomorphism class of $E$ as an $F$-bundle over $B$, but also on the $\rho$ chosen. That "subtlety" won't matter for our purposes...

$\triangleright E$ is holomorphically separable (see [14]).

$\triangleright E$ is Stein if $G_{\text {struct }}(E)$ is compact or contained in a compact group (see [12] and [14]).

$\triangleright E$ is Stein if $G_{\text {struct }}(E)$ has finitely many connected components (see [14]).

Given $g \in \operatorname{Aut}(D)$, there is exactly one bundle $E \stackrel{D}{\longrightarrow} \mathbb{C}^{*}$ with monodromy $g$. Namely, $E=\mathbb{C} \times D / \mathbb{Z}$, where the $\mathbb{Z}$-action is the "diagonal action" generated by

$$
\tilde{g}: \mathbb{C} \times D \longrightarrow \mathbb{C} \times D, \quad(z ; d) \longmapsto(z+1 ; g(d)) .
$$

We shall call that bundle the complex suspension of $g$. It has infinite cyclic $\mathcal{A}(E)$, generated by $g$.

For simplicity, we introduce the following notations and results assuming $n=2$, but they all extend in the "obvious" way to any $n \geq 2$.

By a well-known criterion for the Steinness of a Reinhardt domain (see [8]), the map

$$
\text { 'log' }:\left(\mathbb{C}^{*}\right)^{2} \longrightarrow \mathbb{R}^{2}, \quad\left(z_{1}, z_{2}\right) \longmapsto\left(\log \left|z_{1}\right|, \log \left|z_{2}\right|\right)
$$

induces a one-to-one correspondence between Stein Reinhardt domains of $\left(\mathbb{C}^{*}\right)^{2}$ and open convex subsets of $\mathbb{R}^{2}$.

Now take $D \subset\left(\mathbb{C}^{*}\right)^{2}$ a bounded Stein Reinhardt domain. We shall denote by $\log D$ the image of $D$ by the above map. To make more explicit the one-to-one correspondence

$$
\log D \longleftrightarrow D
$$

remark that $D$ can be recovered from $\log D$ as the image of the "tube" $\log D+i \mathbb{R}^{2} \subset \mathbb{C}^{2}$ by the map

$$
\text { 'exp' : }\left(w_{1}, w_{2}\right) \longmapsto\left(z_{1}, z_{2}\right)=\left(\mathrm{e}^{w_{1}}, \mathrm{e}^{w_{2}}\right) .
$$

Moreover $\log D$ contains no affine line: otherwise $\log D+i \mathbb{R}^{2}$ would contain a copy of $\mathbb{C}$ on which 'exp' would restrict to a non-constant map from $\mathbb{C}$ to $D$, contradicting the boundedness of $D$. By [21], the converse statement holds: for a Stein Reinhardt $D$, if $\log D$ contains no affine line, then $D$ is isomorphic to a bounded domain (we won't use that fact, though).

We denote by $\operatorname{Aut}(D)$ the group of automorphisms of $D$. By [11],

$$
\operatorname{Aut}(D)=\operatorname{Aut}_{\mathrm{alg}}^{\mathbb{R}}(D) \ltimes \operatorname{Aut}_{0}(D),
$$

where

$\triangleright \operatorname{Aut}_{0}(D)$ is the connected component of the identity,

TOME $134-2006-\mathrm{N}^{\mathrm{O}} 4$ 
$\triangleright \operatorname{Aut}_{\text {alg }}(D)$ is the subgroup of $\operatorname{Aut}(D)$ defined by: For each $g \in \operatorname{Aut}_{\text {alg }}(D)$, there exist $A_{g}=\left[\begin{array}{ll}a & b \\ c & d\end{array}\right] \in \mathrm{GL}_{2}(\mathbb{Z})$ and $\alpha_{1}, \alpha_{2} \in \mathbb{C}^{*}$ such that

$$
g\left(z_{1}, z_{2}\right)=\left(\alpha_{1} z_{1}^{a} z_{2}^{b}, \alpha_{2} z_{1}^{c} z_{2}^{d}\right) .
$$

(Given $g$, such $A_{g}, \alpha_{1}$ and $\alpha_{2}$ are unique.)

$\triangleright \operatorname{Aut}_{\mathrm{alg}}^{\mathbb{R}}(D)$ denotes the subgroup of $\operatorname{Aut}_{\mathrm{alg}}(D)$ of all $g$ 's with real and positive $\alpha_{i}$ 's. Thus

$$
\operatorname{Aut}_{\text {alg }}(D)=\operatorname{Aut}_{\text {alg }}^{\mathbb{R}}(D) \ltimes\left(S^{1}\right)^{2},
$$

and by Lemma 1.4, $\operatorname{Aut}_{\text {alg }}^{\mathbb{R}}(D)$ is a discrete group.

For $g \in \operatorname{Autalg}_{\text {alg }}(D)$, we shall denote by $f_{g}$ the map

$$
f_{g}: \log D \longrightarrow \log D, \quad p \longmapsto A_{g} p+b_{g},
$$

where $b_{g}=\left(\log \left|\alpha_{1}\right|, \log \left|\alpha_{2}\right|\right)$. The correspondence $g \leftrightarrow f_{g}$ is one-to-one between $\operatorname{Aut}_{\mathrm{alg}}^{\mathbb{R}}(D)$ and the group of affine automorphisms of $\log D$. Remark that

Define

$$
f_{g^{-1}}(p)=f_{g}^{-1}(p)=A_{g}^{-1} p-A_{g}^{-1} b_{g} .
$$

$$
\mathcal{A}(D)=\left\{A_{g}: g \in \operatorname{Aut}_{\mathrm{alg}}^{\mathbb{R}}(D)\right\} \subset \mathrm{GL}_{2}(\mathbb{Z}) .
$$

It is useful to think of $\mathcal{A}(D)$ as "the set of matrices that act on $D$ ".

For a given bundle $E \stackrel{D}{\longrightarrow} B$,

$$
\mathcal{A}(E)=\left\{A_{g}: g \in \operatorname{Aut}_{\text {alg }}^{\mathbb{R}}(D) \cap\left(\operatorname{Aut}_{0}(D) \cdot G_{\text {struct }}(E)\right)\right\} \subset \mathcal{A}(D) .
$$

It is useful to think of $\mathcal{A}(E)$ as "the set of matrices that are used to build $E$ ".

For any group of matrices $\mathcal{A}$, we denote

$$
\operatorname{Spec}_{\mathbb{C}} \mathcal{A}=\bigcup_{A \in \mathcal{A}} \operatorname{Spec}_{\mathbb{C}} A .
$$

We can now state the main results of this paper. They consist of the following theorems, and the geometric description (that follows from Theorem 1) given below. We point out the importance of the set $\operatorname{Spec}_{\mathbb{C}} \mathcal{A}(D)$ to study whether a domain $D$ belongs to $\mathcal{S}$ or not. Theorem 1 in the case $n=2$ is the main result of [9]. Our proof for that case is simpler.

Theorem 1. - A bounded Stein Reinhardt domain $D \subset \mathbb{C}^{n}$ with $n=2$ or 3 belongs to $\mathcal{S}$ if and only if $\operatorname{Spec}_{\mathbb{C}} \mathcal{A}(D) \subset S^{1}$. In other words:

$\triangleright$ For $n=2, D \notin \mathcal{S}$ if and only if there exists $A \in \mathcal{A}(D)$ with $\operatorname{Spec}_{\mathbb{C}} A=$ $\left\{\lambda, \lambda^{-1}\right\}, \lambda \in \mathbb{R} \backslash\{+1,-1\}$.

$\triangleright$ For $n=3, D \notin \mathcal{S}$ if and only if (cf. Lemma 1.2) there exists $A \in \mathcal{A}(D)$ such that either

(a) $\operatorname{Spec}_{\mathbb{C}} A=\left\{\lambda_{1}, \lambda_{2}, \lambda_{3}\right\}$ with $\lambda_{i}$ 's pairwise distinct and real, or

(b) $\operatorname{Spec}_{\mathbb{C}} A=\left\{1, \lambda, \lambda^{-1}\right\}$ with $\lambda \in \mathbb{R} \backslash\{+1,-1\}$.

BULLETIN DE LA SOCIÉTÉ MATHÉMATIQUe DE FRANCE 
In any manifold $B$, consider a free homotopy class $\gamma: S^{1} \rightarrow B$. By a realanalytic approximation, followed by complexification, $\gamma$ can be realized as a holomorphic map

$$
\{w \in \mathbb{C}: 1<|w|<m\} \longrightarrow B
$$

for some $m \in(1,+\infty]$. We define the holomorphic width of $\gamma$ to be the log of the supremum of all possible such $m$ 's.

Theorem 2. - Let $D \subset \mathbb{C}^{2}$ be a bounded Stein Reinhardt domain. Let $E$ be a bundle over a Stein basis $B$, and with fiber $D$. If $\operatorname{Spec}_{\mathbb{C}} \mathcal{A}(E) \subset S^{1}$ then $E$ is Stein. Conversely, assume that $\operatorname{Spec}_{\mathbb{C}} \mathcal{A}(E) \not \subset S^{1}$, i.e., $B$ contains a homotopy class $\gamma$ with associated monodromy $g \in \operatorname{Aut}(D)$ such that $\rho>1$, where $\rho$ denotes the spectral radius of $A_{g}$. If the holomorphic width of $\gamma$ is strictly greater than $2 \pi^{2} / \log \rho$, then $E$ is not Stein.

Theorem 3. - Let $D \subset\left(\mathbb{C}^{*}\right)^{n}$ be a bounded Stein Reinhardt domain, with $n \geq 2$. Let $E$ be a bundle over a Stein basis, and with fiber D. If

$$
\operatorname{Spec}_{\mathbb{C}} \mathcal{A}(E) \subset S^{1}
$$

then $E$ is Stein.

Theorem 1 naturally leads to the following

Question. - For an arbitrary $n$, does a bounded Stein Reinhardt domain $D \subset \mathbb{C}^{n}$ belong to $\mathcal{S}$ if and only if $\operatorname{Spec}_{\mathbb{C}} \mathcal{A}(D) \subset S^{1}$ ?

We believe the answer to be "yes".

\section{Geometric description of domains not in $\mathcal{S}$}

$\triangleright$ For $\boldsymbol{n}=2$. - It follows from Theorem 1 that if $D \notin \mathcal{S}$, then $D$ has an automorphism $g$ of the form $g\left(z_{1}, z_{2}\right) \mapsto\left(z_{1}^{a} z_{2}^{b}, z_{1}^{c} z_{2}^{d}\right)$ with $A=\left[\begin{array}{ll}a & b \\ c & d\end{array}\right] \in \mathrm{GL}_{2}(\mathbb{Z})$ and $\operatorname{Spec}_{\mathbb{C}} \mathcal{A}(E) \not \subset S^{1}$. Up to replacing $A$ by $A^{2}$, we can assume $A \in \mathrm{SL}_{2}(\mathbb{Z})$ and $\operatorname{Spec}_{\mathbb{C}} A=\left\{\lambda, \lambda^{-1}\right\}, \lambda \in(1,+\infty)$. Then no entry of $A$ is zero, therefore $g$ maps both axes to $(0,0)$, so $D$ must be a subset of $\left(\mathbb{C}^{*}\right)^{2}$. Moreover the action of $g$ on $\left(\mathbb{C}^{*}\right)^{2}$ is well understood:

The eigenspaces of $A$ split $\mathbb{R}^{2}$ into four quadrants, which correspond to four $g$-invariant "quadrant domains" in $\left(\mathbb{C}^{*}\right)^{2}$ that are depicted above. Any quadrant domain is sent isomorphically to a bounded domain by a well-chosen automorphism of $\left(\mathbb{C}^{*}\right)^{2}$. The domain $D_{\mathrm{CL}}$ appearing (as the fiber of a non Stein bundle) in [1] is a quadrant domain of the matrix $\left[\begin{array}{ll}2 & 1 \\ 1 & 1\end{array}\right]$.

Now, boundedness of $D$ implies that $D$ must be contained in a quadrant domain (otherwise $\log D$, being $A$-invariant, would contain an affine line).

Finally we conclude that for $n=2, D \notin \mathcal{S}$ if and only if $D$ is a $g$-invariant Stein subdomain of a quadrant domain of some matrix $A \in \mathrm{SL}_{2}(\mathbb{Z})$.

TOME $134-2006-\mathrm{N}^{\mathrm{O}} 4$ 


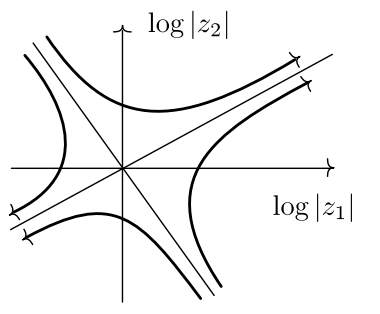

linear action of $A$ on $\mathbb{C}^{2}$

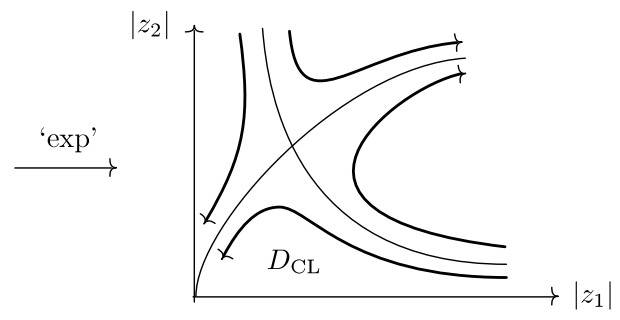

multiplicative action of $g$ on $\left(\mathbb{C}^{*}\right)^{2}$

FiguRe 1

Such $D$ 's are in one-to-one correspondence with convex $A$-invariant open subsets of a quadrant of $A$.

Basic examples are provided by the quadrants domains themselves, and also by taking $D$ such that $\log D$ is the interior of the convex hull of the $A$-orbit of a point in $\mathbb{R}^{2}$ not belonging to an eigenspace of $A$.

For $\boldsymbol{n}=3$. - As opposed to the two-dimensional case, there are examples of domains not in $\mathcal{S}$, but not included in $\left(\mathbb{C}^{*}\right)^{3}$ : the product of the unit disk and any two-dimensional domain not in $\mathcal{S}$ is easily checked not to be in $\mathcal{S}$. Besides those trivial "product examples", domains invariant by a matrix that falls in case (b) of Theorem 1 are some sort of a product with a two-dimensional domain $D^{\prime}$ not in $\mathcal{S}$ (see Section 3.2).

Let $A$ be a matrix in $\mathrm{SL}_{3}(\mathbb{Z})$ satisfying condition (a) of Theorem 1, with eigenvectors $X_{1}, X_{2}$ and $X_{3}$. Examples of domains not in $\mathcal{S}$ are:

1) Any $D$ such that $\log D$ is an octant delimited by the $X_{i}$ 's. This example is the three-dimensional generalization of [1] and [20].

2) Another basic example is given as follows: pick a point $p=x_{1} X_{1}+x_{2} X_{2}+$ $x_{3} X_{3}$ with $x_{1} x_{2} x_{3} \neq 0$. Then take $D$ such that $\log D$ is the interior of the convex hull of $\left\{A^{k} p: k \in \mathbb{Z}\right\}$.

3) In some cases, starting with a $\log D$ from the above example, then reflecting it through a "coordinate plane", then taking the convex hull gives another convex $A$-invariant open subset of $\mathbb{R}^{3}$ that still corresponds to a bounded domain. This produces examples not contained in an octant delimited by the $X_{i}$ 's. These have no counterpart in the case of a twodimensional $D$.

REMARK. - We've given a complete description of all domains not in $\mathcal{S}$ for $n=2$, and a fairly precise description for $n=3$. But a complete answer should also tell when two domains in the list are actually isomorphic. For $n=2$, it actually follows from the above and from the literature about 
Inoue-Hirzebruch surfaces that considering "even Dloussky matrices" (defined in [20]) already yields all examples of $D$ 's not in $\mathcal{S}$.

We shall make use of the following auxiliary results, proved in Section 4:

LEMma 1.1. - The only possible rational eigenvalues for a matrix $A \in \mathrm{GL}_{n}(\mathbb{Z})$ are +1 or -1 .

Lemma 1.2. - Let $A \in \mathrm{GL}_{3}(\mathbb{Z})$. Then exactly one of the following holds:

[1] $\operatorname{Spec}_{\mathbb{C}} A \subset\{+1,-1\}$,

$[2]_{\mathbb{C}} \operatorname{Spec}_{\mathbb{C}} A=\left\{ \pm 1, \lambda, \lambda^{-1}\right\}$ with $\lambda \in \mathbb{C} \backslash\{+1,-1\}$ and $|\lambda|=1$,

$[2]_{\mathbb{R}} \operatorname{Spec}_{\mathbb{C}} A=\left\{ \pm 1, \lambda, \pm \lambda^{-1}\right\}$ with $\lambda \in \mathbb{R} \backslash\{+1,-1\}$,

[3] $\operatorname{Spec}_{\mathbb{C}} A=\left\{\lambda_{1}, \lambda_{2}, \lambda_{3}\right\}$ with $\operatorname{deg}_{\mathbb{Q}} \lambda_{i}=3$ for $i=1,2,3$.

We shall write for short (and that is a disjoint union)

$$
\mathrm{GL}_{3}(\mathbb{Z})=[1] \cup[2]_{\mathbb{C}} \cup[2]_{\mathbb{R}} \cup[3] .
$$

Corollary 1.3. - If $A \in \mathrm{GL}_{3}(Z)$ doesn't have pairwise distinct eigenvalues, then $\operatorname{Spec}_{\mathbb{C}} A \subset\{+1,-1\}$.

LEMMA 1.4. - The set of matrices $\mathcal{A}(D)$ is a subgroup of $\mathrm{GL}_{n}(\mathbb{Z})$, and the map

is a group isomorphism.

$$
\psi: \operatorname{Aut}_{\mathrm{alg}}^{\mathbb{R}}(D) \longrightarrow \mathcal{A}(D), \quad g \longmapsto A_{g}
$$

The following well-known result (see $[4, B(1)(\mathrm{b}), \mathrm{p} .47]$ ) is in some sense a generalization of Bézout's Theorem (which corresponds to the case $n=2$ ).

LEMmA 1.5. - The vector $v=\left[\begin{array}{c}v_{1} \\ \vdots \\ v_{n}\end{array}\right] \in \mathbb{Z}^{n}$ is unimodular $\left(i . e ., \operatorname{gcd}\left(v_{1}, \ldots, v_{n}\right)\right.$ is equal to 1) if and only if there exists a matrix in $\mathrm{SL}_{n}(\mathbb{Z})$ of the form

$$
\left[\begin{array}{cccc}
v_{1} & * & \cdots & * \\
\vdots & \vdots & & \vdots \\
v_{n} & * & \cdots & *
\end{array}\right] .
$$

Lemma 1.6. - Let $D \subset \mathbb{C}^{n}$ be a Stein Reinhardt domain, with $n \geq 2$. Denote

$$
\pi\left(z_{1}, \ldots, z_{n}\right)=\left(z_{2}, \ldots, z_{n}\right) .
$$

Then $\pi(D)$ is a Stein (Reinhardt) domain of $\mathbb{C}^{n-1}$.

LEMMA 1.7. - Let $E \stackrel{D}{\longrightarrow} B$ be a bundle with fiber a Reinhardt domain $D \subset\left(\mathbb{C}^{*}\right)^{n}$. Assume there exists a non-zero $v \in \mathbb{Z}^{n}$ such that $A v=v$ for all $A \in \mathcal{A}(E)$. Then there exists a "quotient bundle"

$$
E^{\prime} \stackrel{D^{\prime}}{\longrightarrow} B
$$

TOME $134-2006-\mathrm{N}^{\mathrm{O}} 4$ 
such that $D^{\prime} \subset\left(\mathbb{C}^{*}\right)^{n-1} ; E^{\prime}$ is Stein if and only if $E$ is Stein; and for any $A^{\prime} \in \mathcal{A}\left(E^{\prime}\right) \subset \mathrm{GL}_{n-1}(\mathbb{Z}), A^{\prime}$ is the automorphism of $\mathbb{Z}^{n} /\langle v\rangle_{\mathbb{Z}} \approx \mathbb{Z}^{n-1}$ induced by some $A \in \mathcal{A}(E)$. In particular,

$$
\operatorname{Spec}_{\mathbb{C}} A\left(E^{\prime}\right) \subset \operatorname{Spec}_{\mathbb{C}} A(E) .
$$

The following result is due to Burnside (cf. [19, p. 33]).

LEMMA 1.8. - Let $V$ be a finite-dimensional vector space over a field $F$. Let $S$ be a semi-group of endomorphisms of $V$ acting irreducibly on $V$. If the elements of $S$ have only finitely many different traces, then $S$ itself is finite.

LEMMA 1.9. - Let $E \stackrel{D}{\longrightarrow} B$ be a flat bundle and $H \subset G_{\text {struct }}(E)$ be a subgroup of finite index. Then there exists a flat bundle

$$
E^{\prime} \stackrel{D}{\longrightarrow} B
$$

with $G_{\text {struct }}\left(E^{\prime}\right)=H$ and such that $E^{\prime}$ is Stein if and only if $E$ is Stein.

Lemma 1.10. - Let $D \subset\left(\mathbb{C}^{*}\right)^{n}$ be a bounded Stein Reinhardt domain, and $E \stackrel{D}{\longrightarrow} B$ be a bundle with $B$ Stein. If $\operatorname{Spec}_{\mathbb{C}} \mathcal{A}(E)$ is finite, then $E$ is Stein.

LEMMA 1.11. - Let $\mathcal{A}$ be a subgroup of $\mathrm{GL}_{n}(Z)$ with $n \geq 2$. If $\operatorname{Spec}_{\mathbb{C}} \mathcal{A} \subset S^{1}$, then $\operatorname{Spec}_{\mathbb{C}} \mathcal{A}$ is finite.

\section{Proof of Theorem 1 for $D \subset \mathbb{C}^{2}$}

The following two results prove Theorem 1 for $n=2$.

Proposition 2.1. - Let $D \subset\left(\mathbb{C}^{*}\right)^{2}$ be a bounded Stein Reinhardt domain. Then $D \in \mathcal{S}$ if and only if $\operatorname{Spec}_{\mathbb{C}} \mathcal{A}(D) \subset S^{1}$.

Proof. — "Only if" part: suppose there exists $A \in \mathcal{A}(D)$ and $\operatorname{Spec}_{\mathbb{C}} A \not \subset S^{1}$. Up to replacing $A$ by $A^{2}$, we can assume that $\operatorname{det} A=1$. As $1 \notin \operatorname{Spec}_{\mathbb{C}} A$, up to conjugation by an automorphism of $\left(\mathbb{C}^{*}\right)^{2}$, we can assume (see p. 460 for more details) that $D$ has an automorphism of the form

$$
g\left(z_{1}, z_{2}\right)=\left(z_{1}^{a} z_{2}^{b}, z_{1}^{c} z_{2}^{d}\right) \quad \text { with } \quad A=\left[\begin{array}{ll}
a & b \\
c & d
\end{array}\right] \in \mathrm{SL}_{2}(\mathbb{Z})
$$

and $\operatorname{Spec}_{\mathbb{C}} A=\left\{\lambda, \lambda^{-1}\right\}, \lambda \neq 1$. Let $E \stackrel{D}{\longrightarrow} \mathbb{C}^{*}$ be the complex suspension of $g$. Then the method of [1] applies almost directly to show that $E$ is not Stein. The only (easy) extra work is to check that $\log D$ contains an $\mathbb{R}$-orbit of the form $\left\{A^{t}\left[\begin{array}{l}x \\ y\end{array}\right]: t \in \mathbb{R}\right\}$ for some $\left[\begin{array}{l}x \\ y\end{array}\right] \in \log D$ (see Section 3 for more details). The proof from [20] can be adapted as well. We conclude that $D \notin \mathcal{S}$.

Conversely, suppose that $\operatorname{Spec} \mathcal{A}(D) \subset S^{1}$. Then by Lemma 1.11, Spec $\mathcal{A}(D)$ is finite. Take a bundle $E \stackrel{D}{\longrightarrow} B$ with $B$ Stein. As $\operatorname{Spec} \mathcal{A}(E) \subset \operatorname{Spec} \mathcal{A}(D)$, $E$ is Stein by Lemma 1.10. This proves that $D \in \mathcal{S}$.

BULletin DE LA SOCiÉtÉ MATHÉmATiQUE DE FRANCE 
Proposition 2.2. - If $D \subset \mathbb{C}^{2}$ with $D \not \subset\left(\mathbb{C}^{*}\right)^{2}$, then $D \in \mathcal{S}$ and $\operatorname{Spec}_{\mathbb{C}} \mathcal{A}(D) \subset S^{1}$.

Proof. - If $D \cap\left\{z_{i}=0\right\}$ is nonempty for $i=1,2$, then by [11, Lemma 1, p. 412], $\operatorname{Aut}_{\text {alg }}^{\mathbb{R}}(D)$ is finite. Therefore $\operatorname{Aut}(D)$ has only finitely many connected components, so $D \in \mathcal{S}$ by [14]. As any $g \in \operatorname{Aut}_{\text {alg }}^{\mathbb{R}}(D)$ has finite order, we also know that $\operatorname{Spec}_{\mathbb{C}} \mathcal{A}(D) \subset S^{1}$.

Else we can assume without loss of generality that $D \cap\left\{z_{1}=0\right\} \neq \varnothing$ and $D \cap\left\{z_{2}=0\right\}=\varnothing$. Then any $g \in \operatorname{Aut}_{\text {alg }}^{\mathbb{R}}(D)$ must have the form $g\left(z_{1}, z_{2}\right)=\left(\alpha_{1} z_{1} z_{2}^{k}, \alpha_{2} z_{2}^{ \pm 1}\right)$. As $D$ is bounded and stable by $g^{n}$ for any $n \in \mathbb{Z}$, we get $\alpha_{2}=1$, so the second component of $g^{n}\left(z_{1}, z_{2}\right)$ has modulus independent from $n$. Now, for the first component to remain bounded for any $\left(z_{1}, z_{2}\right) \in D$, we must have $\alpha_{1}=1$ and $k=0$. $\operatorname{So~}_{\operatorname{Aut}}^{\mathbb{R}}(D)$ is finite and, as above, $D \in \mathcal{S}$ and $\operatorname{Spec}_{\mathbb{C}} \mathcal{A}(D) \subset S^{1}$.

\section{Proof of Theorem 1 for $D \subset \mathbb{C}^{3}$}

3.1. For $D \subset\left(\mathbb{C}^{*}\right)^{3}$, if $\mathcal{A}(D) \subset[1] \cup[2]_{\mathbb{C}}$, then $D \in \mathcal{S}$. - As $\operatorname{Spec}_{\mathbb{C}} \mathcal{A}(D) \subset S^{1}$, it follows from Lemmas 1.11 and 1.10 that $D \in \mathcal{S}$.

3.2. For $D \subset\left(\mathbb{C}^{*}\right)^{3}$, if $\mathcal{A}(D) \cap[2]_{\mathbb{R}} \neq \varnothing$, then $\boldsymbol{D} \notin \mathcal{S}$. - Let $g$ in $\operatorname{Aut}_{\text {alg }}(D)$ be such that $A=A_{g} \in[2]_{\mathbb{R}}$. Up to taking $A^{2}$ instead of $A$, we can assume that $\operatorname{Spec}_{\mathbb{C}} A=\left\{1, \lambda, \lambda^{-1}\right\}$, with $\lambda \in \mathbb{R} \backslash\{+1,-1\}$. As $1 \in \operatorname{Spec}_{\mathbb{Q}} A$, there exists $v \in \mathbb{Q}^{3}$ such that $A v=v$. Up to multiplying by some integer, we can assume that $v \in \mathbb{Z}^{3}$.

Now define $E \stackrel{D}{\longrightarrow} \mathbb{C}^{*}$ as the complex suspension of $g$. Lemma 1.7 applies here and yields a bundle $E^{\prime} \stackrel{D^{\prime}}{\longrightarrow} \mathbb{C}^{*}$ with fiber $D^{\prime} \subset\left(\mathbb{C}^{*}\right)^{2}$, and $\mathcal{A}\left(E^{\prime}\right)$ being generated by $A^{\prime} \in \mathrm{SL}_{2}(\mathbb{Z})$ with $\operatorname{Spec}_{\mathbb{C}} A^{\prime}=\left\{\lambda, \lambda^{-1}\right\}$ and $\lambda \neq \pm 1$. We saw that $E^{\prime}$ is not Stein in the proof of Proposition 2.1. Therefore $D \notin \mathcal{S}$.

3.3. For $D \subset\left(\mathbb{C}^{*}\right)^{3}$, if $\mathcal{A}(D) \cap[3] \neq \varnothing$, then $D \notin \mathcal{S}$. - Let $g$ in $\operatorname{Aut}_{\mathrm{alg}}^{\mathbb{R}}(D)$ be such that $A=A_{g} \in[3]$. We know that $g$ on $D$ corresponds on $\log D$ to

$$
f_{g}:\left[\begin{array}{l}
x_{1} \\
x_{2} \\
x_{3}
\end{array}\right] \longmapsto A\left[\begin{array}{l}
x_{1} \\
x_{2} \\
x_{3}
\end{array}\right]+\left[\begin{array}{l}
b_{1} \\
b_{2} \\
b_{3}
\end{array}\right] .
$$

By Lemma $1.2,1 \notin \operatorname{Spec}_{\mathbb{C}} A$. Therefore $A-I$ is an invertible $3 \times 3$ matrix, and it is easy to check that

$$
p=-(A-I)^{-1}\left[\begin{array}{l}
b_{1} \\
b_{2} \\
b_{3}
\end{array}\right]
$$

is a fixed point of $f_{g}$, and (therefore) the translation $T_{p}: \mathbb{R}^{3} \rightarrow \mathbb{R}^{3}$ of vector $p$ conjugates $f_{g}$ to $A$ (i.e., $T_{p}^{-1} f_{g} T_{p}=A$ ). It follows that up to conjugation by the 
$\left(\mathbb{C}^{*}\right)^{3}$ automorphism $\left(z_{1}, z_{2}, z_{3}\right) \mapsto\left(\mathrm{e}^{p_{1}} z_{1}, \mathrm{e}^{p_{2}} z_{2}, \mathrm{e}^{p_{3}} z_{3}\right)$, we can assume that $g$ is such that $f_{g}\left[\begin{array}{l}x_{1} \\ x_{2} \\ x_{3}\end{array}\right]=A\left[\begin{array}{l}x_{1} \\ x_{2} \\ x_{3}\end{array}\right]$ for any $\left[\begin{array}{l}x_{1} \\ x_{2} \\ x_{3}\end{array}\right] \in \mathbb{R}^{3}$.

Lemma 3.1. - The eigenvalues $\lambda_{1}, \lambda_{2}, \lambda_{3}$ of $A$ are real.

Proof. - As $\mathbb{R}^{3}$ is an odd-dimensional real vector space, we shall assume without loss of generality that $\lambda_{1} \in \mathbb{R}$. We know from Lemma 1.2 that $\lambda_{1} \neq 1$. Up to replacing $A$ by $A^{-1}$, we can assume that $\lambda_{1}>1$.

Now we prove by contradiction that $\lambda_{2}$ is real. Suppose that $\lambda_{2} \in \mathbb{C} \backslash \mathbb{R}$. As $\operatorname{det} A= \pm 1$, we must have $\left|\lambda_{2}\right|<1$. Denote by $X_{1}$ a $\lambda_{1}$-eigenvector, and by $X_{2}$ and $X_{3}$ the imaginary and real parts of a (necessarily complex) $\lambda_{2}$ eigenvector. The matrix of $f_{g}$ with respect to the basis $\left\{X_{1}, X_{2}, X_{3}\right\}$ is

$$
\left[\begin{array}{rrr}
\lambda_{1} & 0 & 0 \\
0 & a & -b \\
0 & b & a
\end{array}\right]
$$

where $a+i b=\lambda_{2}$ (as $\lambda_{2} \notin \mathbb{R}, b$ must be nonzero). As $\log D$ is open, we can pick a point $q=x_{1} X_{1}+x_{2} X_{2}+x_{3} X_{3}$ in $\log D$ with $x_{1} \neq 0$. Then it is not hard to check that the convex hull of $\left\{A^{k} q: k \in \mathbb{Z}\right\}$ (which is in $\log D$ ) must contain the half-space $\left\{a_{1} X_{1}+a_{2} X_{2}+a_{3} X_{3}: a_{1} x_{1}>0\right\}$. This is impossible because $\log D$ contains no affine line. So $\lambda_{2}$ and (therefore) $\lambda_{3}$ are real.

Up to taking $A^{ \pm 2}$ instead of $A$, and renaming the eigenvalues, we can assume that $0<\lambda_{3}<\lambda_{2}<1<\lambda_{1}$.

Let $O^{+}$denote the octant $\left\{\sum_{j} a_{j} X_{j}\right.$ : for all $\left.j, a_{j}>0\right\}$. Again, we shall use coordinates with respect to the basis $\left\{X_{1}, X_{2}, X_{3}\right\}$, namely we write " $\left(p_{1}, p_{2}, p_{3}\right)$ " instead of " $\sum_{j} p_{j} X_{j}$ ".

The next two lemmas help us understand the shape of $\log D$.

LEMMA 3.2. - If $p=\left(p_{1}, p_{2}, p_{3}\right) \in \log D \cap O^{+}$then, for any $t \geq 0$,

$$
\left(t+p_{1}, p_{2}, p_{3}\right) \in \log D \cap O^{+} \quad \text { and } \quad\left(p_{1}, p_{2}, t+p_{3}\right) \in \log D \cap O^{+} .
$$

Proof. - Recall that $0<\lambda_{3}<\lambda_{2}<1<\lambda_{1}$. Denote

$$
\left(x_{n}, y_{n}, z_{n}\right):=A^{n} p=\left(\lambda_{1}^{n} p_{1}, \lambda_{2}{ }^{n} p_{2}, \lambda_{3}{ }^{n} p_{3}\right) .
$$

When $n \rightarrow+\infty, y_{n}$ and $z_{n}$ tend to zero, whereas $x_{n}$ tends to $+\infty$. As a consequence, the angle between the line $\left(p, A^{n} p\right)$ and the line $(p, p+(1,0,0))$ tends to zero. Now take a ball $B$ in $\log D$, centered at $p$. Fix $t \geq 0$. Because of the above facts, the convex hull of $\left\{A^{n} p\right\} \cup B$ will contain $\left(t+p_{1}, p_{2}, p_{3}\right)$ as soon as $n$ is big enough. That proves the first part of the statement.

When $n \rightarrow-\infty, x_{n}$ tends to zero, and $y_{n}$ and $z_{n}$ tend to $+\infty$, with $y_{n} / z_{n}$ tending to zero. Therefore the angle between the line $\left(p, A^{n} p\right)$ and the line $(p, p+(0,0,1))$ tends to zero. The second part of the statement follows as in the above case.

BULLETIN DE LA SOCIÉtÉ MATHÉMATIQUE DE FRANCE 
Lemma 3.3. - There exists $r \in \log D$ such that $\left\{A^{t} r, t \in \mathbb{R}\right\}$ is contained in $\log D$.

Proof. - We shall make repeated use of Lemma 3.2 and of the inequalities $\lambda_{1}>1, \lambda_{2}<1, \lambda_{3}<1$. Take any $q=\left(q_{1}, q_{2}, q_{3}\right) \in \log D$.

$\triangleright$ First case. Assume that $q \in O^{+}$. Then $V_{1}:=\left(\lambda_{1} q_{1}, q_{2}, q_{3}\right)$ is in $\log D$, and therefore $V_{2}:=\left(\lambda_{1} q_{1}, q_{2}, \lambda_{3}{ }^{-1} q_{3}\right)$ is in $\log D$ as well. On the other hand, $A q=\left(\lambda_{1} q_{1}, \lambda_{2} q_{2}, \lambda_{3} q_{3}\right)$ is in $\log D$, so $V_{3}:=\left(\lambda_{1} q_{1}, \lambda_{2} q_{2}, q_{3}\right)$ is in $\log D$, and so is $V_{4}:=\left(\lambda_{1} q_{1}, \lambda_{2} q_{2}, \lambda_{3}{ }^{-1} q_{3}\right)$.

Multiplying the first components by $\lambda_{1}$ we get four other points in $\log D$ : $V_{5}:=\left(\lambda_{1}^{2} q_{1}, q_{2}, q_{3}\right), V_{6}:=\left(\lambda_{1}^{2} q_{1}, q_{2}, \lambda_{3}{ }^{-1} q_{3}\right), V_{7}:=\left(\lambda_{1}{ }^{2} q_{1}, \lambda_{2} q_{2}, q_{3}\right)$, and $V_{8}:=\left(\lambda_{1}^{2} q_{1}, \lambda_{2} q_{2}, \lambda_{3}{ }^{-1} q_{3}\right)$.

Let $r:=V_{2}$. Remark that $V_{7}=A r$. As $\log D$ is convex, it contains the parallelepiped $\mathcal{P}$ with vertices $V_{1}, V_{2}, \ldots, V_{8}$. Thus, if $\lambda_{1} q_{1} \leq x_{1} \leq \lambda_{1}^{2} q_{1}$, $q_{2} \leq x_{2} \leq \lambda_{2} q_{2}$, and $\lambda_{3}{ }^{-1} q_{3} \leq x_{3} \leq q_{3}$, then $\left(x_{1}, x_{2}, x_{3}\right)$ belongs to $\mathcal{P}$, and therefore to $\log D$. It follows that for any $t$ between 0 and $1, A^{t} r=$ $\left(\lambda_{1}{ }^{t} \lambda_{1} q_{1}, \lambda_{2}{ }^{t} q_{2}, \lambda_{3}{ }^{t} \lambda_{3}{ }^{-1} q_{3}\right)$ belongs to $\log D$. As $\log D$ is invariant by $A$, we get that $A^{t} r$ belongs to $\log D$ for any real $t$.

$\triangleright$ Second case. If $q \notin O^{+}$, take a linear automorphism $B$ of $\mathbb{R}^{3}$ such that for all $j, B\left(X_{j}\right)= \pm X_{j}$ (thus $A^{t} B=B A^{t}$ for any $t \in \mathbb{R}$ ), and such that $B q \in O^{+}$. Now apply the first case to get in $B(\log D)$ an $\mathbb{R}$-orbit, which is sent to an $\mathbb{R}$-orbit in $\log D$ by $B^{-1}$.

We now construct a non-Stein bundle $E \stackrel{D}{\longrightarrow} \mathbb{C}^{*}$ by adapting the construction of G. Cœuré and J.-J. Lœb. Lemma 3.3 is an important ingredient for the construction of analytic disks as in [1].

We consider the "tube above $\log D$ ":

$$
V:=\left\{\sum_{j} u_{j} X_{j}:\left(\operatorname{Im} u_{1}, \operatorname{Im} u_{2}, \operatorname{Im} u_{3}\right) \in \log D\right\} \subset \mathbb{C}^{3} .
$$

We consider the action of $G_{\mathbb{R}}=\mathbb{R} \ltimes \mathbb{R}^{3}$ on $\mathbb{C} \times \mathbb{C}^{3}$ given by

$\mathbb{C} \times \mathbb{C}^{3} \times G_{\mathbb{R}} \longrightarrow \mathbb{C} \times \mathbb{C}^{3},\left(\left(z, \sum_{j} u_{j} X_{j}\right),(t, X)\right) \longmapsto\left(t+z, X+\sum_{j} \lambda_{j}^{t} u_{j} X_{j}\right)$.

Define $\Omega:=\mathbb{C} \times V$. By construction, $\Omega$ is invariant by the action of

$$
G_{\mathbb{Z}}:=\mathbb{Z} \ltimes \mathbb{Z}^{3} \subset G_{\mathbb{R}}
$$

Now take $E:=\Omega / G_{\mathbb{Z}}$ (cf. [1]). Remark that $E$ is just the complex suspension of $g$. We shall prove that $E$ is not Stein by constructing a family of analytic disks $\left\{d_{R}: \bar{\Delta} \rightarrow E\right\}_{R>1}$ such that $d_{R}(0)$ tends to infinity in $E$ (topologically) when $R \gg 1$, whereas $d_{R}(\partial \bar{\Delta})$ remains in a compact subset of $E$ independent from $R$. Here $\Delta$ (resp. $\bar{\Delta}$ ) denotes the open (resp. closed) unit disk in $\mathbb{C}$, and $\partial \bar{\Delta}$ denotes their boundary.

TOME $134-2006-\mathrm{N}^{\mathrm{O}} 4$ 
Fix a real number $R>1$. In what follows, it is useful to keep in mind that the maps $f, g_{1}, g_{2}, g_{3}$ and $\tilde{d}$ depend on $R$.

Define $f: \Delta \rightarrow \mathbb{C}$ by

$$
f(z)=\log i \frac{R+z}{R-z} .
$$

Then $0<\operatorname{Im} f<\pi$, and $f$ is continuous on $\bar{\Delta}$.

Thus we can define $g_{1}, g_{2}$ and $g_{3}$ as holomorphic functions $\Delta \rightarrow \mathbb{C}$ such that

$$
\operatorname{Im} g_{j}=\lambda_{j}^{\mu_{1}^{-1} \operatorname{Re} f}
$$

on $\partial \bar{\Delta}$, for $j=1,2,3$, where $\mu_{1}$ denotes $\log \lambda_{1}$.

Define a map $\tilde{d}: \Delta \rightarrow \mathbb{C} \times \mathbb{C}^{3}$ by

$$
\tilde{d}(t):=\left(\mu_{1}^{-1} f(t), \sum_{j} g_{j}(t) r_{j} X_{j}\right),
$$

where $r=\left(r_{1}, r_{2}, r_{3}\right)$ is given by Lemma 3.3. Notice that $\tilde{d}$ is continuous on $\bar{\Delta}$.

Lemma 3.4. - For any $t \in \bar{\Delta}, \tilde{d}(t) \in \Omega$.

Proof. — Recall that $\tilde{d}(t) \in \Omega$ if and only if

$$
\left(r_{1} \operatorname{Im} g_{1}(t), r_{2} \operatorname{Im} g_{2}(t), r_{3} \operatorname{Im} g_{3}(t)\right) \in \log D .
$$

First assume that $t \in \partial \bar{\Delta}$. Denote $\left(\operatorname{Im} g_{1}(t) r_{1}, \operatorname{Im} g_{2}(t) r_{2}, \operatorname{Im} g_{3}(t) r_{3}\right)$ by $\tilde{d}^{\prime}(t)$. By definition of the $g_{j}$ 's, $\tilde{d}^{\prime}(t)=\left(\lambda_{1}^{\alpha} r_{1}, \lambda_{2}^{\alpha} r_{2}, \lambda_{3}^{\alpha} r_{3}\right)$ for some $\alpha \in \mathbb{R}$, thus $\tilde{d}^{\prime}(t)$ is on the $\mathbb{R}$-orbit of $r$, which is in $\log D$ by the Lemma 3.3.

Now for $s \in \Delta$, the componentwise harmonicity of $\tilde{d}^{\prime}$ allows us to write in vector form

$$
\tilde{d}^{\prime}(s)=\int_{S^{1}} \tilde{d}^{\prime}(t) P_{s}(t),
$$

where $P_{s}$ is the Poisson kernel at $s$. As $\int_{S^{1}} P_{s}=1$, the above shows that $\tilde{d}^{\prime}(s)$ is in the convex hull of $\tilde{d}^{\prime}\left(S^{1}\right)$, therefore is in $\log D$.

We define an analytic disk $d_{R}: \Delta \rightarrow E$ by

$$
d_{R}=q_{\mathbb{Z}} \circ \tilde{d},
$$

where $q_{\mathbb{Z}}$ is the quotient map $\Omega \rightarrow E$. Remark that $d$ is continuous on $\bar{\Delta}$.

LEMma 3.5. - There exists a compact set $K$ in $E$, independent from $R$, such that $d_{R}(\partial \bar{\Delta}) \subset K$.

Proof. - It is not hard to check that $\tilde{d}(t)$ is equal up to action by $G_{\mathbb{R}}$ to

$$
\left(i \mu_{1}^{-1} \operatorname{Im} f(t), i \sum_{j} r_{j} \lambda_{j}^{-\mu_{1}^{-1} \operatorname{Re} f(t)} \operatorname{Im} g_{j}(t) X_{j}\right) .
$$

BULLETIN DE LA SOCIÉTÉ MATHÉMATIQUE DE FRANCE 
When $t$ is in $\partial \bar{\Delta}$, the above becomes

$$
\left(i \mu_{1}^{-1} \operatorname{Im} f(t), i \sum_{j} r_{j} X_{j}\right) .
$$

As $0<\operatorname{Im} f<\pi$, there exists a compact set $\widetilde{K} \subset \Omega$ independent from $R$, such that $\tilde{d}(\partial \bar{\Delta}) \subset \widetilde{K} \cdot G_{\mathbb{R}}$. Then $d_{R}(\partial \bar{\Delta})$ is included in $K=q_{\mathbb{Z}}(\widetilde{K}) \cdot\left(G_{\mathbb{R}} / G_{\mathbb{Z}}\right)$, which is compact because $G_{\mathbb{R}} / G_{\mathbb{Z}}$ is compact.

On the other hand,

$$
\tilde{d}(0)=\left(\frac{1}{2} \mu_{1} \pi, \sum_{j} g_{j}(0) r_{j} X_{j}\right),
$$

and $\operatorname{Im} g_{1}(0)=\int_{S^{1}} \operatorname{Im} g_{1}=\int_{S^{1}} \lambda_{1}^{\mu_{1}^{-1} \operatorname{Re} f}=\int_{S^{1}} \mathrm{e}^{\operatorname{Re} f}$, which tends to $+\infty$ when $R$ tends to 1 (cf. [1]).

By construction of $E$, a point $q_{\mathbb{Z}}\left(z ;\left(z_{1}, z_{2}, z_{3}\right)\right)$ in $E$ tends to infinity (topologically) if and only if either $\operatorname{Im} z$ or $\operatorname{Im}\left(z_{1}, z_{2}, z_{3}\right)$ tends to infinity. Therefore when $R$ tends to $1, d_{R}(0)=q_{\mathbb{Z}}(\tilde{d}(0))$ tends to infinity in $E$. By the maximum principle (for functions $\Delta \rightarrow \mathbb{C}$ ), any function on $E$ will be bounded on the sequence $\left\{d_{1+1 / n}(0)\right\}_{n \in \mathbb{N}}$, which proves that $E$ is not holomorphically convex, therefore $E$ is not Stein.

Conclusion: $D \notin \mathcal{S}$.

3.4. The case of $\boldsymbol{D} \not \subset\left(\mathbb{C}^{*}\right)^{3}$. - Up to action on $\mathbb{C}^{3}$ by an algebraic automorphism, we can assume that $D$ is in Shimizu's normal form (in his notations, that means $D=\widetilde{D}$; see [11, p. 410]). Remark: that realization of $D$ may not be bounded.

Proposition 3.6. - If $D \subset \mathbb{C}^{3}$ intersects several coordinate hyperplanes, then $\operatorname{Spec}_{\mathbb{C}} \mathcal{A}(D) \subset S^{1}$ and $D \in \mathcal{S}$.

Proof. $-\triangleright$ First step. If $D \cap z_{i}=0$ is nonempty for $i=1,2$ and 3, then by [11, Lemma 1, p. 412], $\operatorname{Aut}_{\mathrm{alg}}^{\mathbb{R}}(D)$ is finite. Therefore $\operatorname{Aut}(D)$ has only finitely many connected components, and $D \in \mathcal{S}$ by [14]. As any $g \in \operatorname{Aut}_{\text {alg }}^{\mathbb{R}}(D)$ has finite order, we also know that $\operatorname{Spec}_{\mathbb{C}} \mathcal{A}(D) \subset S^{1}$.

$\triangleright$ Second step. Without loss of generality, we can now assume that $D \cap\left\{z_{1}=\right.$ $0\}$ and $D \cap\left\{z_{2}=0\right\}$ are both nonempty and $D \cap\left\{z_{3}=0\right\}$ is empty. (In Shimizu's notations, we must have $\sum_{i=1}^{s} n_{i}=2, t=s+1, n_{t}=1$.)

Take $g \in \operatorname{Aut}_{\mathrm{alg}}^{\mathbb{R}}(D)$. Then $g^{2}$ must be of the form

$$
g^{2}\left(z_{1}, z_{2}, z_{3}\right)=\left(\alpha_{1} z_{1} z_{3}^{\mu_{1}}, \alpha_{2} z_{2} z_{3}^{\mu_{2}}, \alpha_{3} z_{3}\right) .
$$

In particular, $\operatorname{Spec}_{\mathbb{C}} \mathcal{A}(D) \subset S^{1}$. $\operatorname{As} \operatorname{proj}_{3}(D)$ is bounded (in Shimizu's notations, projection of $D$ on $\mathbb{C}^{n_{s+1}} \times \cdots \times \mathbb{C}^{n_{t}}$ is always bounded) and $g^{2}$-invariant, $\alpha_{3}=1$. Now, for any $\left(z_{1}, z_{2}, z_{3}\right) \in D, \operatorname{proj}_{3}^{-1}\left(z_{3}\right)$ is bounded and $g^{2}$-invariant, 
so $\alpha_{1}=\alpha_{2}=1$ and $\mu_{1}=\mu_{2}=0$. It follows that $\operatorname{Aut}_{\text {alg }}^{\mathbb{R}}(D)$ is finite. Therefore, again, $D \in \mathcal{S}$.

We can now assume (until the end of Section 3.4) that $D \cap\left\{z_{1}=0\right\}$ is nonempty, and $D \cap\left\{z_{2}=0\right\}$ and $D \cap\left\{z_{3}=0\right\}$ are both empty.

Then any $g \in \operatorname{Aut}_{\text {alg }}^{\mathbb{R}}(D)$ must be of the form

$$
(*)_{\text {alg }} \quad g\left(z_{1}, z_{2}, z_{3}\right)=\left(\alpha_{1} z_{1} z_{2}^{\mu_{2}} z_{3}^{\mu_{3}}, \alpha_{2} z_{2}^{a} z_{3}^{b}, \alpha_{3} z_{2}^{c} z_{3}^{d}\right),
$$

with $A_{g}^{\prime}=\left[\begin{array}{ll}a & b \\ c & d\end{array}\right] \in \mathrm{GL}_{2}(\mathbb{Z})$. Denote by $\mathcal{A}^{\prime}$ the group

$$
\left\{A_{g}^{\prime} \subset \mathrm{GL}_{2}(\mathbb{Z}): g \in \operatorname{Aut}_{\text {alg }}^{\mathbb{R}}(D)\right\} .
$$

Remark that $\operatorname{Spec}_{\mathbb{C}} \mathcal{A}(D)=\{1\} \cup \operatorname{Spec}_{\mathbb{C}} \mathcal{A}^{\prime}$, so $\operatorname{Spec}_{\mathbb{C}} \mathcal{A}(D) \subset S^{1}$ if and only if $\operatorname{Spec}_{\mathbb{C}} \mathcal{A}^{\prime} \subset S^{1}$.

Proposition 3.7. - If $\operatorname{Spec}_{\mathbb{C}} \mathcal{A}(D) \not \subset S^{1}$, then $D \notin \mathcal{S}$.

Proof. — Take $g \in \operatorname{Aut}_{\text {alg }}^{\mathbb{R}}(D)$ with $\operatorname{Spec}_{\mathbb{C}} A_{g} \not \subset S^{1}$. By $(*)_{\text {alg }}, g$ induces an automorphism $g^{\prime}$ of $D^{\prime}=D \cap\left\{z_{1}=0\right\}$, and $\operatorname{Spec}_{\mathbb{C}} A_{g^{\prime}}^{\prime}=\left\{\lambda, \lambda^{-1}\right\}$ with $\lambda \in \mathbb{R} \backslash\{+1,-1\}$.

Define bundles $E \stackrel{D}{\longrightarrow} \mathbb{C}^{*}$ and $E^{\prime} \stackrel{D^{\prime}}{\longrightarrow} \mathbb{C}^{*}$ as complex suspensions of $g$ and $g^{\prime}$ respectively. The map $D^{\prime} \hookrightarrow D$ induces a map $E^{\prime} \hookrightarrow E$ realizing $E^{\prime}$ as a closed submanifold. By the results of Section 2, we know that $E^{\prime}$ is not Stein, so $E$ can't be Stein.

Conclusion: $D \notin \mathcal{S}$.

Therefore we shall assume until the end of Section 3.4 that

$$
\operatorname{Spec}_{\mathbb{C}} \mathcal{A}(D) \subset S^{1} \text {. }
$$

Our assumptions ( $z_{1}$ vanishes somewhere on $D$ but $z_{2}$ and $z_{3}$ don't) imply by [11] that there can be three cases: (Shimizu's $n_{s+1}, \ldots, n_{t}$ must all be 1 , otherwise by $[11$, p. 411] we would have an $U(2)$-action on $D$, and $D$ would intersect at least two coordinate hyperplanes.)

1) $D$ is bounded, and $\operatorname{Aut}_{0}(D)$ is only $\left(S^{1}\right)^{3}$ (this is Shimizu's $D=\widetilde{D}_{2}$ case).

2) $D=\left\{\left(z_{1}, z_{2}, z_{3}\right): z_{1} \in \mathbb{C},\left(z_{2} \mathrm{e}^{q_{2}\left|z_{1}\right|^{2}}, z_{3} \mathrm{e}^{q_{3}\left|z_{1}\right|^{2}}\right) \in D \cap\left\{z_{1}=0\right\}\right\}$, with $q_{2}, q_{3}$ non-negative real numbers and $q_{2} q_{3} \neq 0$.

3) $D=\left\{\left(z_{1}, z_{2}, z_{3}\right):\left|z_{1}\right|<1,\left(z_{2}\left(1-\left|z_{1}\right|^{2}\right)^{-q_{2} / 2}, z_{3}\left(1-\left|z_{1}\right|^{2}\right)^{-q_{3} / 2}\right) \in D \cap\right.$ $\left.\left\{z_{1}=0\right\}\right\}$, with $q_{2}, q_{3}$ non-negative real numbers (Shimizu's notations for $q_{2}$ and $q_{3}$ are $p_{1}^{2}$ and $p_{1}^{3}$ ).

If $D$ is in case 1$)$. Then any $g$ in Aut $(D)$ has the form $(*)$ alg with $\alpha_{1}, \alpha_{2}, \alpha_{3}$ in $\mathbb{C}^{*}$. Denote $P_{23}\left(z_{1}, z_{2}, z_{3}\right)=\left(z_{2}, z_{3}\right), D_{23}=P_{23}(D)$, and

$$
\widehat{D}=\left\{\left(z_{1}, z_{2}, z_{3}\right): z_{1} \in \mathbb{C},\left(z_{2}, z_{3}\right) \in D_{23}\right\} .
$$

BULlETIN DE LA SOCIÉtÉ MATHÉMATIQUE DE FRANCE 
Take $E \stackrel{D}{\longrightarrow} B$ with Stein $B$. The $\operatorname{Aut}(D)$-action on $D$ induces actions on $\widehat{D}$ and $D_{23}$ such that the sequence of maps $D \hookrightarrow \widehat{D} \rightarrow D_{23}$ is $\operatorname{Aut}(D)$-equivariant. Then we get a sequence of maps of bundles above $B$ :

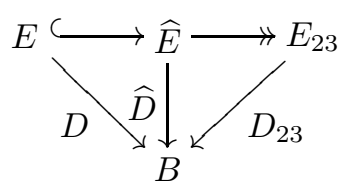

Moreover the map $\widehat{D} \rightarrow D_{23}$ turns $\widehat{D}$ into a (trivial) line bundle over $D_{23}$, and that bundle structure is preserved by the $\operatorname{Aut}(D)$-action on $\widehat{D}$. Therefore $\widehat{E}$ is a line bundle above $E_{23}$.

Now, as $\operatorname{Spec}_{\mathbb{C}} \mathcal{A}\left(E_{23}\right) \subset \operatorname{Spec}_{\mathbb{C}} \mathcal{A}(D) \subset S^{1}$, it follows from Section 2 that $E_{23}$ is Stein. Therefore $\widehat{E}$ is Stein by [7]. By local triviality of our bundles, $E$ is a locally Stein open subset of $\widehat{E}$, so $E$ is Stein by DocquierGrauert Theorem.

Conclusion: $D \in \mathcal{S}$.

If $D$ is in case 2) or 3). The huge formulas in [11, p. 411] tell us that in cases 2) and 3), any $g_{0} \in \operatorname{Aut}_{0}(D)$ is of the form

$$
(*)_{0} \quad g_{0}\left(z_{1}, z_{2}, z_{3}\right)=\left(h_{1}\left(z_{1}\right), z_{2} h_{2}\left(z_{1}\right), z_{3} h_{3}\left(z_{1}\right)\right),
$$

where $h_{1}$ can be any automorphism of $\mathbb{C}$ in case 2 ) and any automorphism of the unit disk in case 3 ), and $h_{2}$ and $h_{3}$ are two holomorphic functions determined by $h_{1}$.

Lemma 3.8. - For any $g \in \operatorname{Aut}_{\mathrm{alg}}^{\mathbb{R}}(D)$, the integers $\mu_{1}$ and $\mu_{2}$ in $(*)_{\text {alg }}$ are both zero.

Proof. — Take $g \in \operatorname{Aut}_{\text {alg }}^{\mathbb{R}}(D)$ (written as in $(*)$ alg $)$, and $g_{0} \in \operatorname{Aut}_{0}(D)$ (written as in $\left.(*)_{0}\right)$ with $h_{1}$ not a homogeneous function. Direct computation yields

$$
\begin{array}{r}
g g_{0} g^{-1}\left(z_{1}, z_{2}, z_{3}\right)=\left(\alpha_{1} h_{1}\left(\alpha_{1}^{-1} z_{1} X\right) X^{-1} h_{2}^{\mu_{2}}\left(\alpha_{1}^{-1} z_{1} X\right) h_{3}^{\mu_{3}}\left(\alpha_{1}^{-1} z_{1} X\right),\right. \\
\left.z_{2} h_{2}^{a+c}\left(\alpha_{1}^{-1} z_{1} X\right), z_{3} h_{3}^{b+d}\left(\alpha_{1}^{-1} z_{1} X\right)\right)
\end{array}
$$

with $X=\alpha_{2}^{\mu_{2} d-\mu_{3} c} \alpha_{3}^{-\mu_{2} b+\mu_{3} a} z_{2}^{-\mu_{2} d+\mu_{3} c} z_{3}^{\mu_{2} b-\mu_{3} a}$. As $\operatorname{Aut}_{0}(D)$ is a normal subgroup of $\operatorname{Aut}(D), g g_{0} g^{-1}$ belongs to $\operatorname{Aut}_{0}(D)$, so the above expression must be of the form $(*)_{0}$. In particular, the first component of $g g_{0} g^{-1}\left(z_{1}, z_{2}, z_{3}\right)$ does not depend on $z_{2}$ or $z_{3}$. As $h_{1}$ is not homogeneous, $X$ itself must be independent of $z_{2}$ or $z_{3}$. Therefore $\mu_{2}=\mu_{3}=0$.

Now $(*)$ alg can be stated more precisely as

$$
(*)_{\mathrm{alg}}^{\prime} \quad g\left(z_{1}, z_{2}, z_{3}\right)=\left(\alpha_{1} z_{1}, \alpha_{2} z_{2}^{a} z_{3}^{b}, \alpha_{3} z_{2}^{c} z_{3}^{d}\right) .
$$

TOME $134-2006-\mathrm{N}^{\mathrm{O}} 4$ 
As $\operatorname{Spec}_{\mathbb{C}} \mathcal{A}(D) \subset S^{1}, \operatorname{Spec}_{\mathbb{C}} \mathcal{A}^{\prime} \subset S^{1}$. Therefore, by Lemmas 1.11 and 1.8, there are two possibilities:

1) $\mathcal{A}^{\prime}$ is finite. Then by $(*)_{\text {alg }}^{\prime}, \mathcal{A}(D)$ is finite, so $D \in \mathcal{S}$ by [14].

2) There exists $v=(p, q) \in \mathbb{Q}^{2}$ such that for any $A^{\prime} \in \mathcal{A}^{\prime}, A^{\prime} v=v$.

Up to division or multiplication by some integer, we can assume that $v$ belongs to $\mathbb{Z}^{2}$ and is unimodular. By Lemma 1.5, there exists $\left(p^{\prime}, q^{\prime}\right)$ such that $\left[\begin{array}{cc}p & p^{\prime} \\ q & q^{\prime}\end{array}\right]$ belongs to $\mathrm{SL}_{2}(\mathbb{Z})$. Consider the automorphism

$$
h: \mathbb{C} \times\left(\mathbb{C}^{*}\right)^{2} \longrightarrow \mathbb{C} \times\left(\mathbb{C}^{*}\right)^{2}, \quad\left(z_{1}, z_{2}, z_{3}\right) \longmapsto\left(z_{1}, z_{2}^{p} z_{3}^{p^{\prime}}, z_{2}^{q} z_{3}^{q^{\prime}}\right) .
$$

We replace $D$ with $h(D)$. The automorphisms get conjugated by $h$, so any $g_{0}$ and $g$ in $\operatorname{Aut}_{0}(D)$ and $\operatorname{Aut}_{\text {alg }}^{\mathbb{R}}(D)$ now take the forms

$$
\begin{array}{cl}
(*)_{0}^{\prime} & g_{0}\left(z_{1}, z_{2}, z_{3}\right)=\left(h_{1}\left(z_{1}\right), z_{2} h_{2}^{q^{\prime}} h_{3}^{-p}\left(z_{1}\right), z_{3} h_{2}^{-q} h_{3}^{p}\left(z_{1}\right)\right), \\
(*)_{\text {alg }}^{\prime \prime} & g\left(z_{1}, z_{2}, z_{3}\right)=\left(\alpha_{1} z_{1}, \alpha_{2} z_{2}, \alpha_{3} z_{3} z_{2}^{\star}\right) .
\end{array}
$$

Take a bundle $E \stackrel{D}{\longrightarrow} B$ with $B$ Stein. Denote $P_{12}\left(z_{1}, z_{2}, z_{3}\right)=\left(z_{1}, z_{2}\right)$, $P_{1}\left(z_{1}, z_{2}\right)=z_{1}, D_{12}=P_{12}(D), D_{1}=P_{1}\left(D_{12}\right)$ and

$$
\begin{aligned}
\widehat{D} & =\left\{\left(z_{1}, z_{2}, z_{3}\right):\left(z_{1}, z_{2}\right) \in D_{12}, z_{3} \in \mathbb{C}\right\}, \\
\widehat{D}_{12} & =\left\{\left(z_{1}, z_{2}\right): z_{1} \in D_{1}, z_{2} \in \mathbb{C}\right\} .
\end{aligned}
$$

These domains are Stein by Lemma 1.6.

The forms $(*)_{0}^{\prime}$ and $(*)_{\text {alg }}^{\prime \prime}$ show that we get an induced $\operatorname{Aut}(D)$-action on $D_{12}, D_{1}, \widehat{D}$ and $\widehat{D}_{12}$ such that the sequence of maps

$$
D \hookrightarrow \widehat{D} \rightarrow D_{12} \hookrightarrow \widehat{D}_{12} \rightarrow D_{1}
$$

is equivariant. So we get a corresponding sequence of maps of bundles above $B$ :

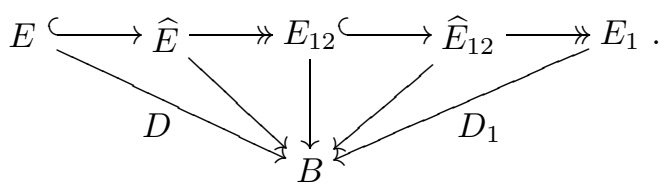

In analogy with case 1) above, we see that $\widehat{E}$ and $\widehat{E}_{12}$ are line bundles above $E_{12}$ and $E_{1}$ respectively. Now, as $D_{1}$ is one dimensional, $E_{1}$ is Stein by [7]. The same reasoning as in case 1 ) shows that $E$ is Stein.

Conclusion: $D \in \mathcal{S}$.

\section{Postponed proofs}

Proof of Theorem 2. - We already proved that if $\operatorname{Spec}_{\mathbb{C}} \mathcal{A}(E) \subset S^{1}$, then $E$ is Stein for $D$ of dimension 2 or 3 during the proof Theorem 1. 
Conversely, assume that there exist $\varepsilon_{1}>0$, an annulus $U \subset \mathbb{C}$ of modulus $m=2 \pi^{2} / \log \rho+\varepsilon_{1}$ and $\varphi: U \rightarrow B$ with associated monodromy $g \in \operatorname{Aut}(D)$, such that $\rho>1$, where $\rho$ denotes the spectral radius of $A_{g}$. As $\operatorname{Spec}_{\mathbb{C}} A_{g} \notin S^{1}$, it follows by Propodition 2.2 that $D \subset\left(\mathbb{C}^{*}\right)^{2}$ and that $D$ is an $A_{g}$-invariant subdomain of a "quadrant domain" (or "Cœuré-Lœb's domain"; cf. Introduction).

Then the pulled-back bundle $E^{\prime}=\varphi^{*}(E)$ has monodromy $g$, therefore $E^{\prime}$ is just the restriction to the annulus $U$ of the complex suspension bundle of $g$ (i.e., Cœuré-Lœb's bundle built from the matrix $A_{g}$ ).

The universal cover of $U$ can be realized as

$$
\left\{w \in \mathbb{C}:-\varepsilon_{2}<\operatorname{Im} w<\frac{\pi}{\log \rho}+\varepsilon_{2}\right\}
$$

with deck automorphism $w \mapsto w+1$ and $\varepsilon_{2}>0$. As noticed in Proposition 2.1, the method of [1] can be directly adapted to the bundle $E^{\prime}$. One gets a sequence of holomorphic disks $d_{n}^{\prime}: \Delta \rightarrow E^{\prime}$ whose boundaries are contained in a compact $K$ independent of $n$, and such that $d_{n}^{\prime}(0) \rightarrow \infty$ topologically in $E^{\prime}$. Moreover, there is a fiber of $E^{\prime}$ that contains $d_{n}^{\prime}(0)$ for all $n$.

Take a bundle map $\tilde{\varphi}: E^{\prime} \hookrightarrow E$ over $\varphi$ that restricts to an isomorphism on each fiber. The holomorphic disks in $E$ defined by $d_{n}=\tilde{\varphi} \circ d_{n}^{\prime}$ have boundaries in the compact set $\tilde{\varphi}(K)$, and centers that tend to infinity. This shows that $E$ is not Stein.

Proof of Theorem 3. - The theorem follows from Lemmas 1.11 and 1.10.

Proof of Lemma 1.1. - Let $\lambda \in \mathbb{Q} \cap \operatorname{Spec}_{\mathbb{C}} A$. Denote by $P_{A}$ the characteristic polynomial of $A$. Then $P_{A}(\lambda)=0$, but as $P_{A}$ is monic and has integer coefficients, any rational root of $P_{A}$ must be in $\mathbb{Z}$. Thus $\lambda \in \mathbb{Z}$. As $\lambda^{-1} \in$ $\operatorname{Spec}_{\mathbb{C}} A^{-1}$, we obtain similarly $\lambda^{-1} \in \mathbb{Z}$. Therefore $\lambda=+1$ or $\lambda=-1$.

Proof of Lemma 1.2. - Denote $\operatorname{Spec}_{\mathbb{C}} A=\left\{\lambda_{1}, \lambda_{2}, \lambda_{3}\right\}$. Let $P_{A}$ be the characteristic polynomial of $A$. Let $P_{\lambda_{1}} \in \mathbb{Q}[X]$ be the minimal polynomial of $\lambda_{1}$ over $\mathbb{Q}$.

$\triangleright$ If $\operatorname{deg} P_{\lambda_{1}}=1$, then $\lambda_{1} \in \mathbb{Q}$, so by Lemma $1.1, \lambda_{1}= \pm 1$. Therefore $P_{A}$ can be written (*) $P_{A}=\left(X-\lambda_{1}\right) Q$ with $Q \in \mathbb{Q}[X]$ and $\operatorname{deg} Q=2$.

- If $\lambda_{2}= \pm 1, \operatorname{det} A=1$ implies $\lambda_{3}= \pm 1$ and we are in case [1].

- If $\lambda_{2} \neq \pm 1$. Then $\lambda_{2} \notin \mathbb{Q}$, and by $\left({ }^{*}\right), Q\left(\lambda_{2}\right)=0$ (remark also that necessarily $\operatorname{deg}_{\mathbb{Q}} \lambda_{2}=2$ and $\left.\operatorname{deg}_{\mathbb{Q}} \lambda_{3}=2\right)$. Denoting $\lambda=\lambda_{2}$, $\operatorname{det} A= \pm 1$ gives

$$
\operatorname{Spec}_{\mathbb{C}} A=\left\{\lambda_{1}= \pm 1, \lambda_{2}=\lambda, \lambda_{3}= \pm \lambda^{-1}\right\} .
$$

If $\lambda \in \mathbb{R}$, we are in case $[2]_{\mathbb{R}}$. If $\lambda \in \mathbb{C} \backslash \mathbb{R}$, then $\lambda_{3}$ must equal $\bar{\lambda}_{2}$ (as complex roots of the real polynomial $P_{A}$ ). As $\lambda_{3}=-1 / \lambda$ is impossible

TOME $134-2006-\mathrm{N}^{\mathrm{O}} 4$ 
(it would imply $\lambda \bar{\lambda}=-1$ ), we get $\lambda_{3}=\lambda^{-1}$, which implies $\lambda \bar{\lambda}=1$, so we are in case $[2]_{\mathbb{C}}$.

$\triangleright$ If $\operatorname{deg} P_{\lambda_{1}}=2$, then $\lambda_{1} \notin \mathbb{Q}$. As $P_{A}\left(\lambda_{1}\right)=0$, one can write $P_{A}=P_{\lambda_{1}} R$ with $R \in \mathbb{Q}[X]$ and $\operatorname{deg} R=1$. Therefore $P_{A}$ has a root in $\mathbb{Q}$, which must be \pm 1 . The same arguments as above show that we are in cases $[2]_{\mathbb{C}}$ or $[2]_{\mathbb{R}}$, and that $\operatorname{deg}_{\mathbb{Q}} \lambda_{i} \leq 2$ for $i=1,2,3$.

$\triangleright$ If $\operatorname{deg} P_{\lambda_{1}}=3$, then $\operatorname{deg}_{\mathbb{Q}} \lambda_{i}=3$ for $i=1,2,3$ because none of the above cases can occur (there we had $\operatorname{deg}_{\mathbb{Q}} \lambda_{i} \leq 2$ for $i=1,2,3$ ). So we are in case [3].

Proof of Lemma 1.3. - Suppose that $\lambda$ is a multiple root of $P_{A}$. Assume $A$ falls in case [3]. Then as $P_{A}^{\prime}(\lambda)=0$ and $\operatorname{deg} P_{A}^{\prime}=2$, we get that $\operatorname{deg}_{\mathbb{Q}} \lambda \leq 2$, which is impossible.

When $A$ falls in case $[2]_{\mathbb{C}}$ or $[2]_{\mathbb{R}}$, it is easy to check that the condition $\lambda \neq \pm 1$ prevents $P_{A}$ from having a multiple root. Therefore $A$ falls in case [1].

Proof of Lemma 1.6. - This lemma is proved, as a particular case, in the next proof.

Proof of Lemma 1.7. - Up to dividing by some integer, we can assume that $v$ is unimodular. The matrix $M$ given by Lemma 1.5 yields an algebraic automorphism $h$ of $\left(\mathbb{C}^{*}\right)^{n}$ by the usual correspondence. Now, up to conjugation by $h$, we can assume that any $g \in G_{\text {struct }}(E)$ is of the form

$$
g\left(z_{1}, \ldots, z_{n}\right)=\left(\alpha_{1} z_{1} z_{2}^{*} \cdots z_{n}^{*}, \alpha_{2} z_{2}^{*} \cdots z_{n}^{*}, \ldots \ldots, \alpha_{n} z_{2}^{*} \cdots z_{n}^{*}\right) .
$$

Therefore the $S^{1}$-action on $D$,

$$
S^{1} \times D \longrightarrow D, \quad\left(\theta ;\left(z_{1}, \ldots, z_{n}\right)\right) \longmapsto\left(\theta z_{1}, \ldots, z_{n}\right),
$$

commutes with the structural group $G_{\text {struct }}(E)$, so we get an induced $S^{1}$-action on $E$.

On the other hand, the projection map

$$
\pi: D \longrightarrow\left(\mathbb{C}^{*}\right)^{n-1}, \quad\left(z_{1}, \ldots, z_{n}\right) \longmapsto\left(z_{2}, \ldots, z_{n}\right)
$$

is $G_{\text {struct }}(E)$-invariant, with the action on $\pi(D)$ being defined by

$$
g\left(\pi\left(z_{1}, \ldots, z_{n}\right)\right)=\pi\left(g\left(z_{1}, \ldots, z_{n}\right)\right) .
$$

Therefore $\pi: D \rightarrow D^{\prime}=\pi(D)$ induces a bundle map $q$ :

$$
\frac{\widetilde{B} \times D}{\pi_{1}(B)}=E \longrightarrow E_{B}^{\prime}=\frac{\widetilde{B} \times D^{\prime}}{\pi_{1}(B)}
$$

BULletin DE LA SOCiÉtÉ MATHÉMATIQUE DE FRANCE 
Now consider the following equivalence relation on $E: x \sim y$ when $f(x)=$ $f(y)$ for all $S^{1}$-invariant holomorphic functions on $E$. Then by [3] we know that $E / \sim$ is a complex space, which is Stein if $E$ is Stein.

We now prove that $q: E \rightarrow E^{\prime}$ is a realization of that quotient, i.e., $x \sim y$ if and only if $q(x)=q(y)$.

$\triangleright$ If $q(x) \neq q(y)$ then as $E^{\prime}$ is holomorphically separable (cf. Section 1), there exists $f \in \mathcal{O}\left(E^{\prime}\right)$ such that $f(q(x)) \neq f(q(y))$. Then $f \circ q \in \mathcal{O}(E)$ separates $x$ and $y$. By construction, the $S^{1}$-orbits are contained in the fibers of $q$, so $f \circ q$ is $S^{1}$-invariant. Thus $x \not y$.

$\triangleright$ If $q(x)=q(y)$ then $x$ and $y$ both belong to the same fiber of $q$, which is a one-dimensional annulus (connectedness of that fiber follows from the convexity of $\left.\log \left(D \cap\left(\mathbb{C}^{*}\right)^{n}\right)\right)$.

Any $S^{1}$-invariant function on $E$ is constant on each circle of that annulus, hence constant on the whole annulus (by the isolated zeros theorem). Thus $x \sim y$.

We still need to show that if $E^{\prime}$ is Stein, then so is $E$. Assume $E^{\prime}$ is Stein. Extending the $G_{\text {struct }}(E)$-action from $D$ to

$$
\widehat{D}=\left\{\left(z_{1}, \ldots, z_{n}\right): z_{1} \in \mathbb{C}^{*},\left(z_{2}, \ldots, z_{n}\right) \in D^{\prime}\right\},
$$

we get an equivariant map $D \hookrightarrow \widehat{D}$, and (therefore) a corresponding injective bundle map:

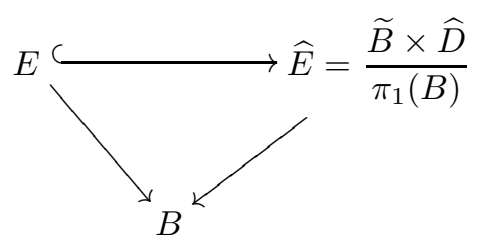

As $\widehat{E}$ is a $\mathbb{C}^{*}$-principal bundle over $E^{\prime}$, it is Stein by the theorem of Matsushima and Morimoto. By local triviality of our bundles, $E$ is locally Stein in $\widehat{E}$. Therefore $E$ is Stein by the Docquier-Grauert Theorem.

Proof of Lemma 1.4. - Take $A_{g}$ and $A_{g^{\prime}}$ in $\mathcal{A}(D)$. It is easy to check that $A_{g g^{\prime}}$ is just $A_{g} A_{g^{\prime}}$. The inverse matrix $A_{g}^{-1}$ comes from $g^{-1}$ by the above remark. Therefore $\mathcal{A}(D)$ is a group and $\psi$ is a morphism, which is onto by definition of $\mathcal{A}(D)$. Moreover, $\psi$ is one-to-one.

Assume $A_{g}=A_{g^{\prime}}$. Then

$$
f_{g}^{-1} f_{g^{\prime}}(p)=f_{g}^{-1}\left(A_{g^{\prime}} p+b^{\prime}\right)=A_{g}^{-1} A_{g^{\prime}} p+A_{g}^{-1} b^{\prime}-A_{g} b=p+A_{g}^{-1}\left(b^{\prime}-b\right) .
$$

If $b^{\prime}-b \neq 0$, then $A_{g}^{-1}\left(b^{\prime}-b\right) \neq 0$, so $f_{g}^{-1} f_{g^{\prime}}$ is a non-trivial translation of $\mathbb{R}^{3}$ which induces an automorphism of $\log D$. This is impossible because $\log D$ contains no affine line.

Thus $b^{\prime}=b$. That proves the injectivity of $\psi$.

TOME $134-2006-\mathrm{N}^{\mathrm{O}} 4$ 
Proof of Lemma 1.9. - As $E$ is flat, it is given by a morphism $\rho: \pi_{1}(B) \rightarrow$ $\operatorname{Aut}(D)$ (see p. 453). Take $G:=\rho^{-1}(H)$. Then the bundle

$$
E^{\prime}=(\widetilde{B} \times D) / G \stackrel{D}{\longrightarrow} B
$$

has $G_{\text {struct }}\left(E^{\prime}\right)=H$ and is Stein if and only if $E$ is Stein, because it is a finite cover of $E$.

Proof of Lemma 1.10. - We know by [11] that any $g \in G_{\text {struct }}(E)$ has the form

$$
g\left(z_{1}, \ldots, z_{n}\right)=\left(\alpha_{1} z_{1}^{a_{11}} \ldots z_{n}^{a_{1 n}}, \ldots, \alpha_{n} z_{1}^{a_{n 1}} \ldots z_{n}^{a_{n n}}\right)
$$

with $\left(a_{i j}\right)_{1 \leq i, j \leq n} \in \mathcal{A}(E) \subset \mathrm{GL}_{n}(\mathbb{Z})$ and $\left(\alpha_{1}, \ldots, \alpha_{n}\right) \in\left(\mathbb{C}^{*}\right)^{n}$.

As $\operatorname{Spec}_{\mathbb{C}} \mathcal{A}(E)$ is finite, is follows from Lemma 1.8 that either $\mathcal{A}(E)$ is finite, or there exists an $\mathcal{A}(E)$-stable $\mathbb{Q}$-subspace $\{0\} \subsetneq V_{1} \subsetneq \mathbb{Q}^{n}$. In the latter case, call $\mathcal{A}_{1} \subset \mathrm{GL}\left(V_{1}\right)$ the group of linear automorphisms induced by $\mathcal{A}(E)$. Being contained in $\operatorname{Spec}_{\mathbb{C}} \mathcal{A}(E), \operatorname{Spec}_{\mathbb{C}} \mathcal{A}_{1}$ is finite, so we can apply Burnsides' result again to $\mathcal{A}_{1}$ acting on $V_{1}$. We repeat that procedure until we get a positive-dimensional subspace $V_{k} \subsetneq \mathbb{Q}^{n}$ such that $\mathcal{A}_{k}$ is finite (notice that if $\operatorname{dim} V_{k}=1$, then finiteness of $\operatorname{Spec}_{\mathbb{C}} \mathcal{A}_{k}$ implies finiteness of $\mathcal{A}_{k}$ ). Then a finite index subgroup of $\mathcal{A}(E)$ (namely, the kernel of $\mathcal{A}(E) \rightarrow \mathcal{A}_{k}$ ) fixes $V_{k}$ pointwise. By Lemma 1.9, we can assume that $\mathcal{A}(E)$ fixes $V_{k}$ pointwise.

Pick $v \in V_{k} \cap \mathbb{Z}^{n}$. Lemma 1.7 applies and yields a bundle $E^{\prime} \stackrel{D^{\prime}}{\longrightarrow} B$ with

- $E^{\prime}$ is Stein if and only if $E$ is,

- $\operatorname{dim} D^{\prime}=n-1$ and $D^{\prime}$ is Stein by Lemma 1.6,

- any $g \in G_{\text {struct }}\left(E^{\prime}\right)$ still has the form (*) (with $n-1$ variables),

- $\operatorname{Spec}_{\mathbb{C}} \mathcal{A}\left(E^{\prime}\right)$ is a subset of $\operatorname{Spec}_{\mathbb{C}} \mathcal{A}(E)$, and is therefore finite.

Even though $D^{\prime}$ may be unbounded, the above properties allow us to repeat the procedure from the beginning. This sets up an induction on $n$, so we only need to prove the theorem for $n=1$. But in that case, as the fiber is of dimension one, $E$ is Stein by [7].

Proof of Lemma 1.11. - Assume $\operatorname{Spec}_{\mathbb{C}} \mathcal{A} \subset S^{1}$. We prove finiteness of the set of all characteristic polynomials of all matrices in $\mathcal{A}$.

Take any $A \in \mathcal{A}$, and let $P$ be its characteristic polynomial. By assumption, all roots of $P$ have modulus 1 . As $\operatorname{deg} P=n, P$ has $n+1$ coefficients. Each of them is an integer of modulus not greater than $n$, because it is given by an elementary symmetric expression in the roots of $P$. Therefore the set of polynomials has cardinal at most $(n+1)^{2 n+1}$.

Conclusion: $\operatorname{Spec}_{\mathbb{C}} \mathcal{A}$ is finite. 


\section{BIBLIOGRAPHY}

[1] CeEuré (G.) \& LeB (J.-J.) - A counterexample to the Serre problem with a bounded domain of $\mathbb{C}^{2}$ as fiber, Ann. of Math., t. 122 (1985), pp. 329-334.

[2] Diederich (K.) \& Fornaess (J.) - Pseudoconvex domains: bounded strictly plurisubharmonic exhaustion functions, Invent. Math., t. 39 (1977), pp. 129-141.

[3] Heinzner (P.) - Kompakte Transformationsgruppen Steinscher Räume (Compact transformation groups of Stein spaces), Math. Ann., t. 285 (1989), pp. 13-28.

[4] Hellegouarch (Y.) - Invitation to the mathematics of Fermat-Wiles, Academic Press, Inc., San Diego, CA, 2002.

[5] Hirschowitz (A.) - Domaines de Stein et fonctions holomorphes bornées, Math. Ann., t. 213 (1975), pp. 185-193.

[6] KAuP (W.) - Reelle Transformationsgruppen und invariante Metriken auf komplexen Räume, Invent. Math., t. 3 (1967), pp. 43-70.

[7] Mok (N.) - Le problème de Serre pour les surfaces de Riemann, C. R. Acad. Sci. Paris Sér. A-B, t. 290 (1980), pp. A179-A180.

[8] Narasimhan (R.) - Several complex variables, Chicago Lectures in Math., University of Chicago Press, Chicago, IL, 1995.

[9] Pflug (P.) \& ZwoneK (W.) - The Serre problem with Reinhardt fibers, Ann. Inst. Fourier, t. 54 (2004), pp. 129-146.

[10] SERRE (J.-P.) - Quelques problèmes globaux relatifs aux variétés de Stein, in Colloque sur les fonctions de plusieurs variables, Bruxelles, 1953, pp. 57 68.

[11] Shimizu (S.) - Automorphisms of bounded Reinhardt domains, Japan. J. Math. (N.S.), t. 15 (1989), pp. 385-414.

[12] Sibony (N.) - Fibrés holomorphes et métrique de Carathéodory, C. R. Acad. Sci. Paris Sér. A, t. 279 (1974), pp. 261-264.

[13] Siu (Y. T.) - All plane domains are Banach-Stein, Manuscripta Math., t. 14 (1974), pp. 101-105.

[14] _ Holomorphic fiber bundles whose fibers are bounded Stein domains with zero first Betti number, Math. Ann., t. 219 (1976), pp. 171-192.

[15] , Pseudoconvexity and the problem of Levi, Bull. Amer. Math. Soc., t. 84 (1978), pp. 481-512.

[16] Skoda (H.) - Fibrés holomorphes à base et à fibre de Stein, Invent. Math., t. 43 (1977), pp. 97-107.

[17] StehlÉ (J.-L.) - Fonctions plurisousharmoniques et convexité holomorphe de certains fibrés analytiques, C. R. Acad. Sci. Paris Sér. A, t. 279 (1974), pp. 235-238.

[18] SteIn (K.) - Überlagerungen holomorph-vollständiger komplexer Räume, Arch. Math., t. 7 (1956), pp. 354-361. 
[19] Steinberg (R.) - Conjugacy classes in algebraic groups, Lecture Notes in Math., vol. 366, Springer-Verlag, Berlin-New York, 1974, notes by V. V. Deodhar.

[20] Zaffran (D.) - Serre problem and Inoue-Hirzebruch surfaces, Math. Ann., t. 319 (2001), pp. 395-420.

[21] ZwoneK (W.) - On hyperbolicity of pseudoconvex Reinhardt domains, Arch. Math. (Basel), t. 72 (1999), pp. 304-314. 